\title{
CCNB2, NUSAP1 and TK1 are associated with the prognosis and progression of hepatocellular carcinoma, as revealed by co-expression analysis
}

\author{
LINGLONG LIU, ANNING CHEN, SIYU CHEN, WEI SONG, QINGMEI YAO, PENGFEI WANG and SUFANG ZHOU \\ Department of Biochemistry and Molecular Biology, School of Pre-Clinical Science, \\ Guangxi Medical University, Nanning, Guangxi 530021, P.R. China
}

Received August 10, 2019; Accepted January 10, 2020

DOI: $10.3892 /$ etm.2020.8522

\begin{abstract}
The mortality rate associated with hepatocellular carcinoma (HCC) is the third highest among all digestive system tumors. However, the causes of HCC development and the underlying mechanisms have remained to be fully elucidated. In the present bioinformatics study, genetic markers were identified and their association with HCC was determined. The mRNA expression datasets GSE87630, GSE74656 and GSE76427 were downloaded from the Gene Expression Omnibus (GEO) database. A total of 96 differentially expressed genes (DEGs) were screened from the 3 GEO datasets, including 25 upregulated and 71 downregulated genes. DEGs were uploaded to the database for Annotation, Visualization and Integrated Discovery to screen for enriched Gene Ontology terms in various categories and the Search Tool for the Retrieval of Interacting Genes/Proteins was used to identify the interactions and functions of the DEGs. A total of 3 genetic markers were identified in a stepwise pathway and functional analysis in a previous study. The association of the genetic markers with prognosis was analysed using the UALCAN online analysis tool. Regression analysis was also performed to identify the relationship between $\mathrm{HCC}$ grade and disease recurrence and the expression of genetic markers using The Cancer Genome Atlas HCC dataset. In addition, the expression of the 3 genetic markers in HCC tissues was determined using reverse transcription-quantitative PCR, the Oncomine database and the Human Protein Atlas database. The expression levels of the 3 genetic markers cyclin B2 (CCNB2), nucleolar and spindle-associated protein 1 (NUSAP1) and thymidine kinase 1 (TK1) were significantly correlated with each other
\end{abstract}

Correspondence to: Dr Sufang Zhou, Department of Biochemistry and Molecular Biology, School of Pre-Clinical Science, Guangxi Medical University, 22 Shuangyong Road, Nanning, Guangxi 530021, P.R. China

E-mail: zsf200000@163.com

Key words: hepatocellular carcinoma, hub genes, biological marker, gene expression profiling, prognosis and high mRNA expression of CCNB2 was significantly associated with poor overall survival of patients with HCC. Receiver operating characteristic curve analysis indicated that NUSAP1 and TK1 were capable of distinguishing between recurrent and non-recurrent HCC. Furthermore, CCNB2, NUSAP1 and TK1 were highly correlated with the HCC grade. It was also indicated that the mRNA expression of CCNB2, NUSAPA and TK1 was increased in primary HCC tissues when compared with that in adjacent tissues. The present study identified that the CCNB2, NUSAP1 and TK1 genes may serve as prognostic markers for HCC, and may be of value from the perspectives of basic research and clinical treatment of HCC.

\section{Introduction}

Liver cancer is one of the most common malignancies of the digestive system (1) and the fourth most frequent cause of cancer-associated mortality worldwide. The most common liver cancer type is hepatocellular carcinoma (HCC) (2). Since HCC lacks reliable clinical and biochemical features in the early stages, the majority of patients are only definitively diagnosed with $\mathrm{HCC}$ at an advanced stage. At present, in addition to hepatectomy, combination therapy also has a significant role in HCC treatment, but a suitable and effective curative treatment strategy is yet to be developed. Of note, $70 \%$ of patients with HCC exhibit tumor recurrence or metastasis within 5 years of receiving the currently applied curative treatment (3). The majority of HCCs occur in patients with underlying liver disease, mostly as a result of hepatitis B or $\mathrm{C}$ virus infection and/or alcohol abuse (4-6). Numerous risk factors associated with poor prognosis and recurrence of HCC have been identified, including histological grade, regional invasion and distant metastasis $(7,8)$. In the last decade, with the wide application of genome-wide gene expression chips, several molecular markers have been detected based on gene expression profiles, several of which are used in the clinical treatment of HCC. These markers have proven valuable for early diagnosis, molecular typing, sensitivity to chemotherapy and drug resistance, prediction of prognosis and monitoring.

Several pathways involved in the generation and development of HCC have been identified, including the 
PI3K/Akt, mitogen-activated protein kinase/ERK (9-11), Janus kinase 2/STAT3 $(12,13)$ and Wnt/ $\beta$-catenin signaling pathways (14). However, numerous molecular mechanisms of hepatocarcinogenesis remain elusive. In the present study, genetic markers and their clinical value in HCC were identified based on data from the Gene Expression Omnibus (GEO) database and The Cancer Genome Atlas (TCGA), and gene expression was then analyzed in association with HCC prognosis and progression.

\section{Materials and methods}

Differentially expressed genes (DEGs) from the GEO database. The mRNA expression datasets GSE87630, GSE74656 and GSE76427 were downloaded from the GEO database (https://www.ncbi.nlm.nih.gov/geo/). The GSE87630 dataset consisted of $64 \mathrm{HCC}$ and 30 non-tumor samples (Illumina HumanHT-12 V3.0 expression BeadChip), the GSE74656 dataset consisted of 5 HCC and 5 non-tumor samples (GeneChip ${ }^{\circledR}$ PrimeView ${ }^{\mathrm{TM}}$ Human Gene Expression Array) and the GSE76427 dataset consisted of 115 HCC and 52 non-tumor samples (Illumina HumanHT-12 V4.0 expression BeadChip). Calculations for each dataset were performed using the GEO2R online analysis tools (https://www.ncbi. nlm.nih.gov/geo/geo2r/). Genes with an adjusted $\mathrm{P}<0.05$ and $\mid \log _{2}$ (fold change) $\mid \geq 1$ were considered differentially expressed. The 'Roubust Rank Aggreg' R package was used to screen the list of DEGs from the 3 microarray datasets obtained using the GEO2R online analysis tools. The list of up- or downregulated DEGs in the 3 chips was used for subsequent analysis.

Functional and pathway analysis of DEGs. DEGs were uploaded to the Database for Annotation, Visualization and Integrated Discovery (https://david.ncifcrf.gov/) for analysis of gene ontology (GO) terms, including GO functional analysis [categories: Biological processes (BP), cellular component (CC) and molecular function (MF)] and Kyoto Encyclopedia of Genes and Genomes (KEGG) pathways. Terms with $\mathrm{P}<0.05$ were considered staistically significant.

Candidate gene selection. The Search Tool for the Retrieval of Interacting Genes/Proteins (version 11.0; https://string-db.org/) is a system used to identify the interactions and functional associations between DEGs. The protein-protein interaction (PPI) was visualized using Cytoscape 3.5.1 (https://cytoscape.org/) to create integrated networks. The CytoHubba plugin was used to screen for hub genes using the maximal clique centrality (MCC) algorithm. In addition, together with document and enrichment analysis, the hub genes that may be interrelated with other hub genes and took part in the same pathway as the genetic markers were selected and used for further analysis and validation.

Survival analysis and clinical features of genetic markers. The expression levels of genetic markers were validated using the Oncomine database (https://www.oncomine.org/) and the Human Protein Atlas (HPA) database (http://www. proteinatlas.org/) (15). Prognostic analysis was performed for genetic markers using the UALCAN online analysis tool (http://ualcan.path.uab.edu/index.html) (16), which incorporates the prognostic data from the TCGA database. Patients with low gene expression (75\%) were assigned to the low/medium expression group and those with high gene expression (25\%) were categorized into the high expression group. A Kaplan-Meier curve for the OS of patients with HCC from the TCGA was obtained according to the low and high expression of each gene. Regression analysis was also performed to identify the clinical features of the genetic markers using TCGA data. Gene expression correlation analysis was used to determine the correlation between genetic marker expression and HCC staging. Spearman's method was used to calculate the correlation coefficient and the datasets, including normal tissue and cancer tissue at different stages were used for analysis. The receiver operating characteristic (ROC) curve was plotted and the area under the curve (AUC) was calculated with SPSS 22.0 (IBM Corp.) to evaluate the capability of genetic markers in distinguishing recurrent and non-recurrent HCC using TCGA data.

Reverse transcription-quantitative $(R T-q) P C R$. A total of $16 \mathrm{HCC}$ and 16 paired adjacent non-tumor tissues were obtained following surgeries performed between August 2016 and September 2018 at the Oncology Affiliated Hospital of Guangxi Medical University (Guangxi, China). Informed consent was obtained from all patients prior to use of their tissues. The present study was approved by the Ethics Committee of Guangxi Medical University (Guangxi, China) and in accordance with the Guangxi Medical University ethical guidelines and regulations. Total RNA was extracted from tissues using the E.Z.N.A. FFPE RNA Isolation kit (Omega Bio-Tek, Inc.). Complementary (c)DNA was obtained using the PrimeScript RT-qPCR kit (Takara Bio, Inc.) according to the manufacturer's protocol. PCR amplification was performed using the GoTaq qPCR Master Mix with SYBR green I (Takara Bio, Inc.). The mRNA expression level was normalized to GAPDH. The primer sequences for cyclin B2 (CCNB2), nucleolar and spindle-associated protein 1 (NUSAP1) and thymidine kinase 1 (TK1) and GAPDH were as follows: CCNB2 forward, 5'-ATGCGTGCCATCCTAGTG GA-3' and reverse, 5'-CGGGAAACTGGCTGAACCTG-3'; NUSAP1 forward, 5'-GGTGCAAGACTGTCCGTGTGG-3' and reverse, 5'-TGGTGCTCGTCTGGTGGAGAAG-3'; TK1 forward, 5'-GTTCTCAGGAAAAAGCACAGAG-3' and reverse, 5'-GTCTTTGGCATACTTGATCACC-3'; GAPDH forward, 5'-AGGTCGGTGTGAACGGATTTG-3' and reverse, 5'-GGGGTCGTTGATGGCAACA-3' (Sangon Biotech Co., Ltd.). The PCR thermocycling conditions were as follows: Initial denaturation at $95^{\circ} \mathrm{C}$ for $30 \mathrm{sec}$ and 40 cycles of $95^{\circ} \mathrm{C}$ for $5 \mathrm{sec}$ and $60^{\circ} \mathrm{C}$ for $34 \mathrm{sec}$. For each sample, the experiment was performed in triplicate in a $20-\mu 1$ reaction volume containing $1 \mu 1$ diluted cDNA, $8 \mathrm{pmol} / \mu 1$ forward and reverse primers, $10 \mu \mathrm{l} \mathrm{SYBR}$ Premix Ex Taq ${ }^{\mathrm{TM}}$ (Takara Bio, Inc.) and $0.4 \mu 1$ ROX II Reference Dye (Takara Bio, Inc.). The relative expression of CCNB2, NUSAP1 and TK1 to GAPDH was calculated using the $2^{-\triangle \Delta \mathrm{Cq}}$ method (17).

Statistical analysis. Statistical analysis was performed using R-3.5.3 and SPSS 22.0 (IBM Corp.). The $\chi^{2}$ test and Fisher's exact test were performed to compare count data and a Student's t-test was performed to compare continuous 
A

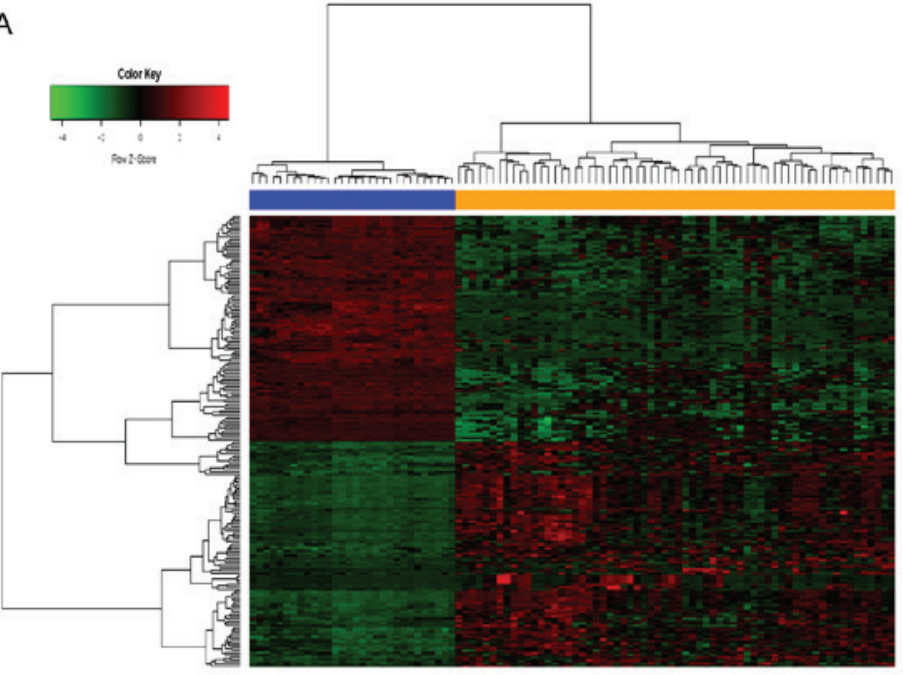

C

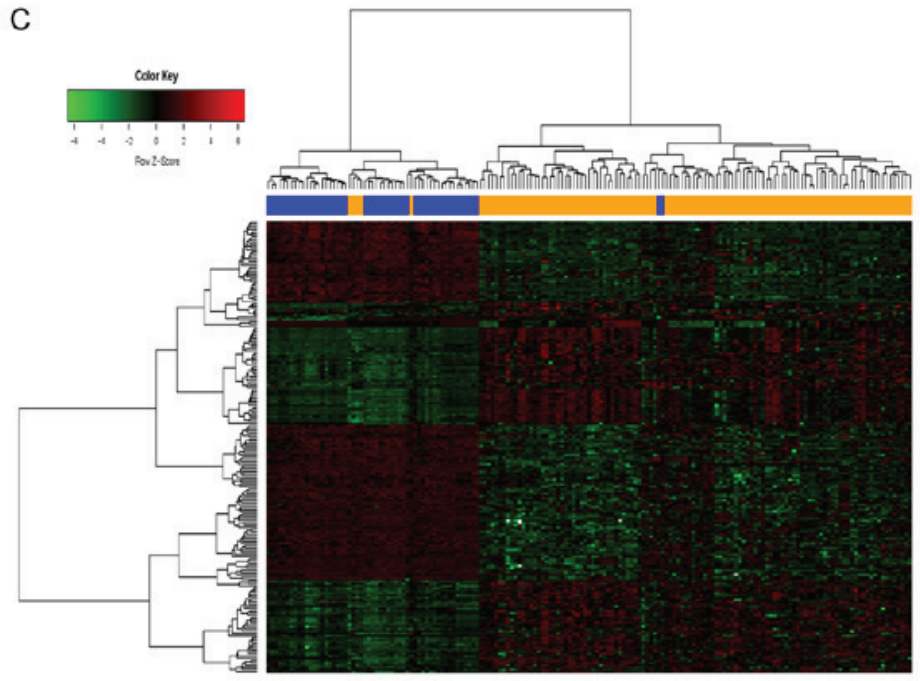

Figure 1. Hierarchical clustering heatmaps of differentially expressed genes screened on the basis of $|\mathrm{FC}|>2.0$ and a corrected P-value $<0.05$. Heatmaps generated using the datasets (A) GSE87630, (B) GSE74656 and (C) GSE76427 are provided. The intensity of the color scheme is scaled to expression values $\left(\log _{2} \mathrm{FC}\right)$, which are Z-score standardized per gene. The color bar above the heatmap represents the sample groups, with orange indicating the tumor sample and blue representing normal samples. FC, fold change.

data between groups. Kaplan-Meier curves were drawn and log-rank tests were performed to assess patient survival. $\mathrm{P}<0.05$ was considered to indicate a statistically significant difference.

\section{Results}

Screening of DEGs. The HCC expression microarray datasets were processed using the GEO2R online analysis tool to obtain DEGs. A total of 1,163 DEGs were screened from the GSE87630 dataset, including 395 up- and 768 downregulated genes. The cluster heatmap of the top 100 up- and downregulated genes is presented in Fig. 1A. A total of 971 DEGs were screened from the GSE74656 dataset, including 556 up- and 414 downregulated genes. The cluster heatmap of the top 100 up- and downregulated genes is provided in Fig. 1B. A total of 494 DEGs were screened from the GSE76427 dataset, including 92 up- and 402 downregulated genes. The cluster heatmap of the top 100 up- and downregulated genes
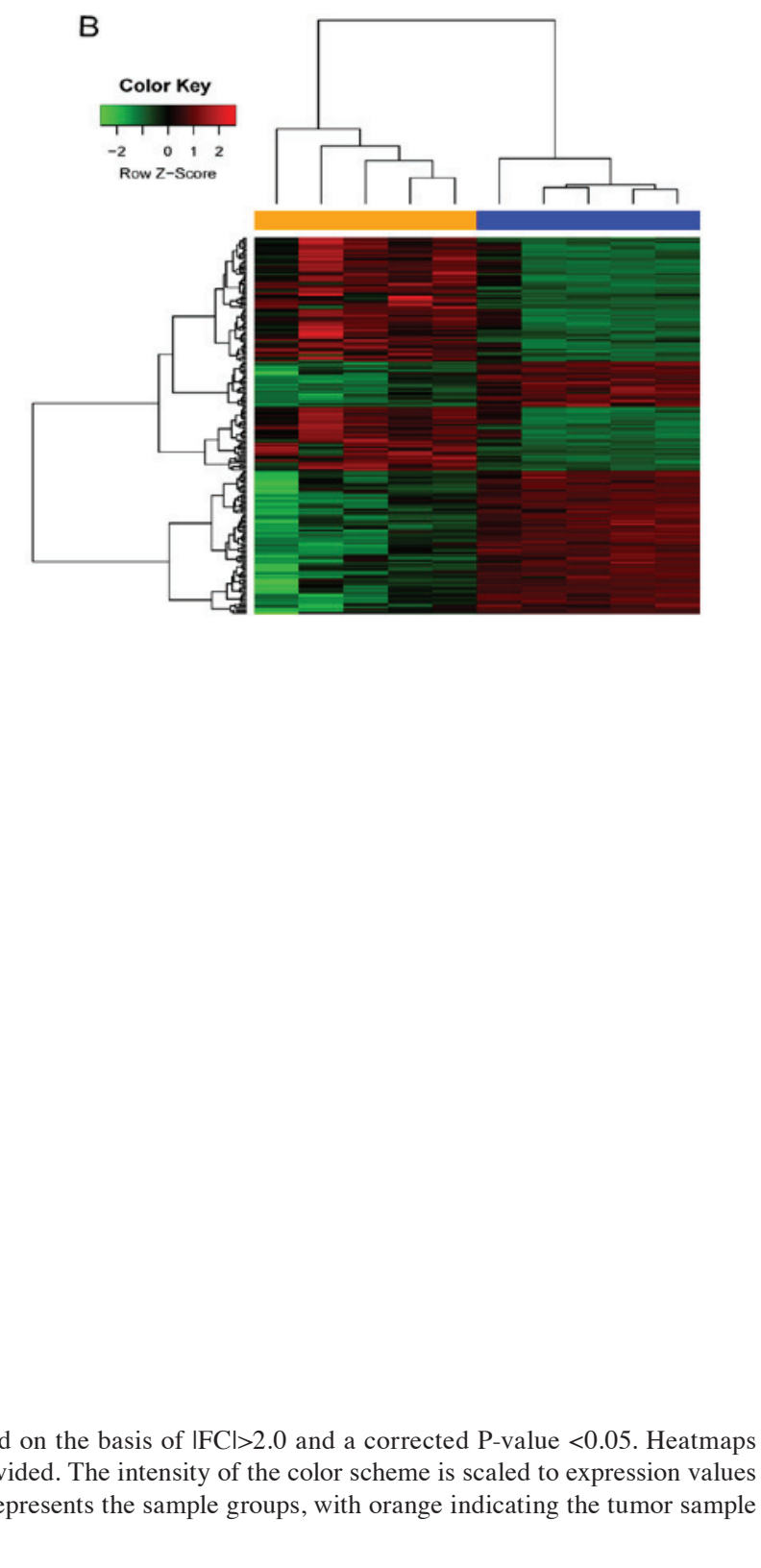

is presented in Fig. 1C. Using the robust rank aggregation (RRA) method (corrected $\mathrm{P}<0.05$ ), 96 integrated DEGs were identified, including 25 up- and 71 downregulated genes. The heatmap of the top 20 up- and downregulated integrated DEGs is provided in Fig. 2.

DEG enrichment analysis. Significant terms and pathways from the GO enrichment and KEGG analysis of DEGs in HCC are presented in Tables I and II, respectively, and in Fig. 3A and B, respectively. The significantly enriched GO terms in the category BP were 'anaphase-promoting complex-dependent catabolic process', 'oxidation-reduction process' and 'cell division'. The significantly enriched GO terms in the category MF were 'transmembrane signaling receptor activity', 'enzyme binding' and 'serine-type endopeptidase activity'. The significantly enriched GO terms in the category CC were 'brush border membrane', 'spindle' and 'organelle membrane' (Fig. 3A). Furthermore, the KEGG analysis revealed the highest accumulation of the DEGs in 'tryptophan metabolism' and 'metabolic pathways'. The 


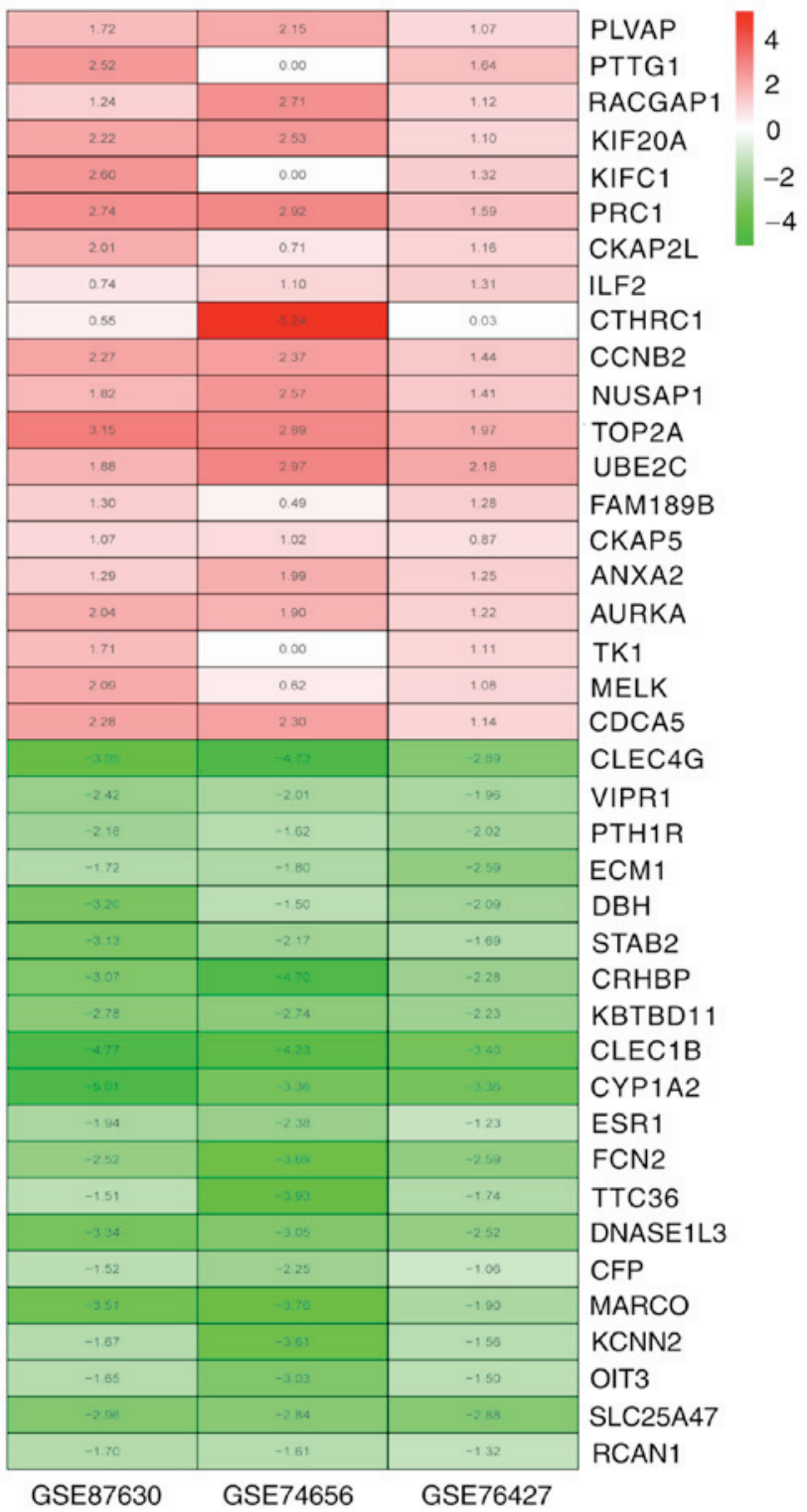

Figure 2. Common differential genes in each expression microarray (GSE87630, GSE74656 and GSE76427). Red represents upregulated genes, green represents downregulated genes.

present results indicated that DEGs were significantly enriched in cell division and metabolic pathways (Fig. 3B).

PPI network analysis of DEGs. A total of 96 DEGs, including 25 up- and 71 downregulated genes, were used to construct a PPI network, as presented in Fig. 4A. The top 15 hub genes were screened according to their MCC values and the hub genes identified were aurora kinase (AURKA), cell division cycle (CDC20), DNA topoisomerase II $\alpha$ (TOP2A), CCNB2, kinesin family member (KIFC1), protein regulator of cytokinesis (PRC1), KIF20A, Rac GTPase-activating protein 1 (RACGAP1), PTTG1, ubiquitin-conjugating enzyme (UBE2C), maternal embryonic leucine zipper kinase (MELK), NUSAP1, cytoskeleton-associated protein 2 like (CKAP2L) and TK1. Among the 15 hub genes, CCNB2, NUSAP1 and TK1 were indicated to have a close association with each other. In a previous study using integration of GRNInfer with GO, KEGG, BioCarta, GNF-U133A, UNIGENE-EST,
Table I. Top 30 Gene Ontology terms of differentially genes associated with hepatocellular carcinoma.

A, Biological process

\begin{tabular}{lrc}
\hline Term & Count & P-value \\
\hline $\begin{array}{l}\text { Anaphase-promoting complex-dependent } \\
\text { catabolic process }\end{array}$ & 5 & 0.000818 \\
Oxidation-reduction process & 11 & 0.001144 \\
Cell division & 8 & 0.002569 \\
Cell surface receptor signaling pathway & 7 & 0.003351 \\
Cellular response to cadmium ion & 3 & 0.003587 \\
G2/M transition of mitotic cell cycle & 5 & 0.006063 \\
Methylation & 4 & 0.006851 \\
Xenobiotic metabolic process & 4 & 0.008223 \\
Mitotic nuclear division & 6 & 0.010251 \\
Mitotic cytokinesis & 3 & 0.010277
\end{tabular}

$\mathrm{B}$, Cellular component

\begin{tabular}{lrc}
\hline Term & Count & P-value \\
\hline Brush border membrane & 5 & 0.000127 \\
Spindle & 6 & 0.000368 \\
Organelle membrane & 5 & 0.000983 \\
Collagen trimer & 5 & 0.001211 \\
Midbody & 5 & 0.004142 \\
Microtubule & 7 & 0.004918 \\
Extracellular region & 17 & 0.006435 \\
Extracellular space & 15 & 0.007454 \\
Extracellular exosome & 24 & 0.011258 \\
Spindle pole & 4 & 0.017791 \\
\hline
\end{tabular}

C, Molecular function

\begin{tabular}{lcc}
\hline Term & Count & P-value \\
\hline $\begin{array}{l}\text { Transmembrane signaling receptor } \\
\text { activity }\end{array}$ & 6 & 0.005480 \\
$\begin{array}{l}\text { Enzyme binding } \\
\text { Serine-type endopeptidase activity }\end{array}$ & 7 & 0.008315 \\
Carbohydrate binding & 5 & 0.011224 \\
Microtubule binding & 5 & 0.020020 \\
Oxygen binding & 3 & 0.024267 \\
Heme binding & 4 & 0.035824 \\
Oxidoreductase activity, acting on paired & 3 & 0.036374 \\
donors, with incorporation or reduction & & \\
of molecular oxygen & & \\
Pyridoxal phosphate binding & 3 & 0.036374 \\
Calcium-dependent protein binding & 3 & 0.037546 \\
\hline
\end{tabular}

Disease and GenMAPP databases by DAVID and MAS 3.0, a corresponding BRCA1 and E2F transcription factor 1 (E2F1) feedback-interactive network as constructed 
Table II. Kyoto Encyclopedia of Genes and Genomes pathway analysis of differentially expressed genes associated with hepatocellular carcinoma.

\begin{tabular}{lrrl}
\hline Pathway & Count & P-value & \\
\hline hsa04114: Oocyte meiosis & 7 & 0.000085 & ADCY1, CCNB2, CPEB3, AURKA, CAMK2B, CDC20, PTTG1 \\
hsa00380: Tryptophan metabolism & 5 & 0.000134 & AADAT, GCDH, KMO, CYP1A2, INMT \\
hsa01100: Metabolic pathways & 15 & 0.024348 & AADAT, GCDH, CYP2C19, NAT2, CYP26A1, KMO, CYP1A2, \\
& & & DBH, TK1, ACSM3, CSAD, HAO2, PEMT, ABAT, DEGS1 \\
hsa00232: Caffeine metabolism & 2 & 0.033000 & NAT2, CYP1A2 \\
hsa00983: Drug metabolism-other & 3 & 0.037585 & NAT2, TPMT, TK1
\end{tabular}
enzymes

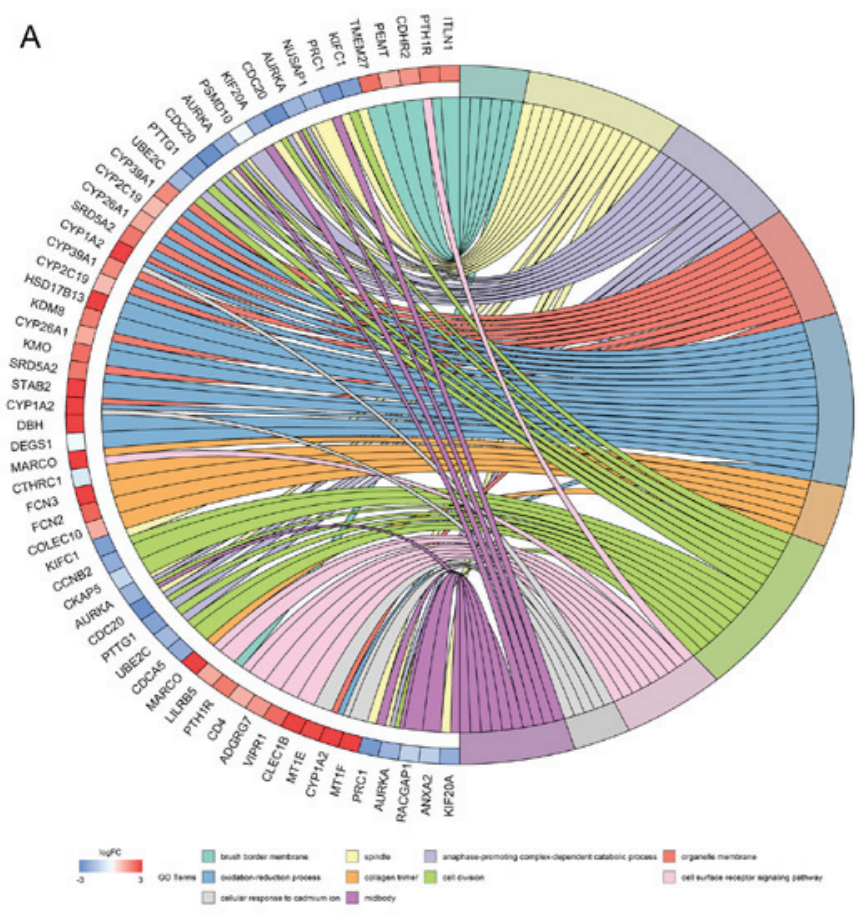

B

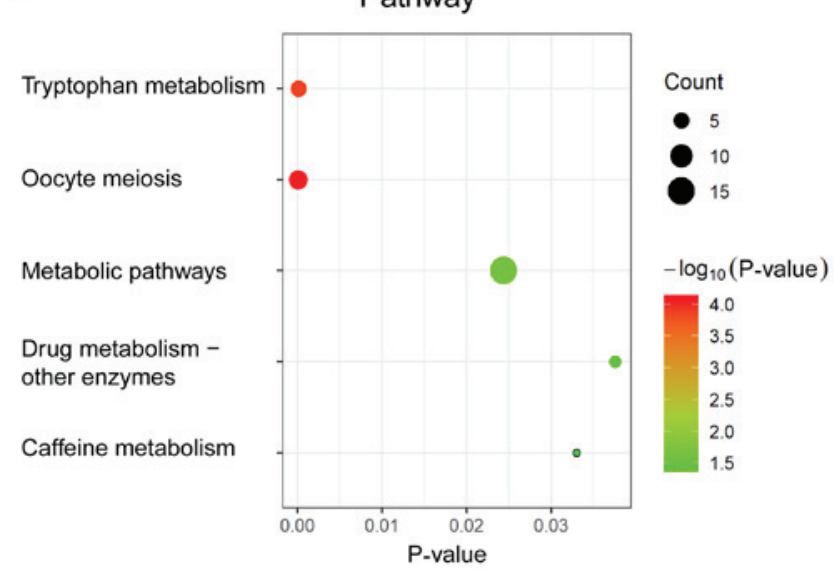

Figure 3. Enrichment analysis of DEGs in HCC. (A) GO enrichment significance items of DEGs in different functional groups. (B) Kyoto Encyclopedia of Genes and Genomes pathway enrichment analysis for DEGs in HCC. FC, fold change; HCC, hepatocellular carcinoma; DEG, differentially expressed gene; GO, gene ontology. and CCNB2-NUSAP1 was indicated to be activated upstream and TK1 was located downstream in HCC (18). In the present study, CCNB2, NUSAP1 and TK1 were identified as genetic markers with involvement of E2F1 and interacting with the BRCA1 pathway in HCC, and were also indicated to be closely associated with other hub genes (Fig. 4B).

Prognostic potential and correlation analysis of 3 genetic markers in HCC. Based on the TCGA data, it was investigated whether any correlation was present between the expression of CCNB2 and NUSAP1 or TK1. The results indicated that the 3 genetic markers had a moderately or highly positive correlation; the expression of CCNB2 and NUSAP1 was highly correlated, while the expression of TK1 was moderately correlated with that of CCNB2 and NUSAP1 ( $<<0.05$; Fig. 5A-C).

The influence of the expression of CCNB2, NUSAP1 and TK1 on survival and of patients with HCC was also analyzed. The expression of CCNB2 was revealed to be positively associated with survival. Higher expression of CCNB2 was associated with poorer survival, while the expression of NUSAP1 and TK1 was not associated with survival. A slight trend for high expression was associated with poor prognosis ( $\mathrm{P}<0.05$; Fig. 5D-F).

Regression analysis was performed to determine the association between the expression of CCNB2, NUSAP1 or TK1 and the histopathological grade. The results suggested that the expression of each of the 3 genetic markers exhibited a mild positive correlation with the histopathological grade (Fig. 5G-I).

ROC curve analysis was used to assess the ability of the expression levels of CCNB2, NUSAP1 and TK1 to distinguish between patients with recurrent and not-recurrent HCC. The results suggested that AUC values of NUSAP1 and TK1 were $>0.5(\mathrm{P}<0.05)$, whereas $\mathrm{CCNB} 2$ had no significant potential to distinguish between patients with recurrent and non-recurrent HCC (Fig. 5J-L). However, the AUCs in the present study are relatively small $(\sim 0.56)$ and may not be sufficient for each molecule on its own to predict recurrence. Hence, they may be used in combination with other predictive factors.

Expression levels of genetic markers in HCC tissue. To investigate the clinical significance of the expression of the genetic markers identified in $\mathrm{HCC}$, the expression of 

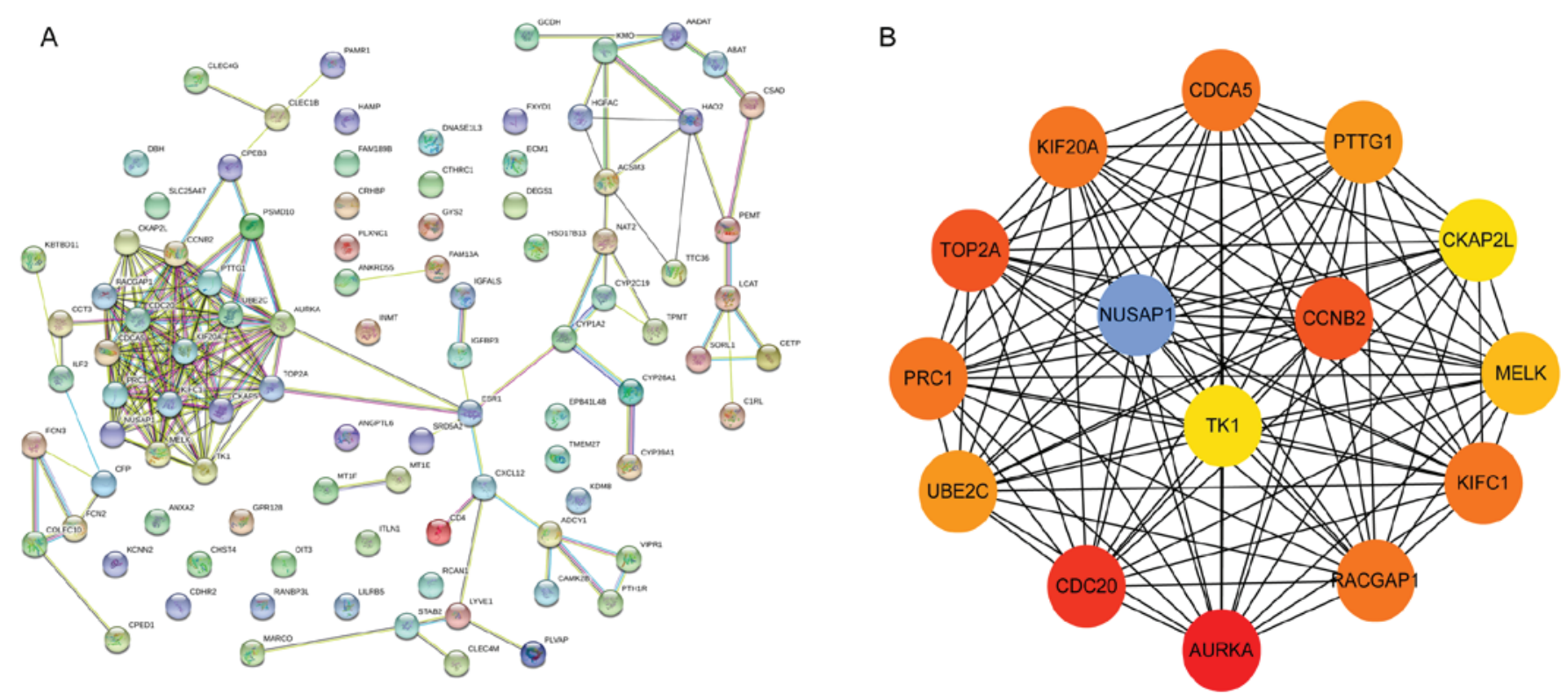

Figure 4. (A) PPI network. Circles represent genes, lines represent the interaction of proteins between genes and the results within the circle represent the structure of proteins. Line colors indicate the interaction between the proteins. (B) Visualization of the 15 hub genes selected from the PPI network using the maximal clique centrality algorithm and the cytoHubba plugin. Edges represent the protein-protein associations. Red octagons represent DEGs with the PPI scores. Yellow octagons represent DEGs with low PPI scores. DEG, differentially expressed gene; PPI, protein-protein interaction.

CCNB2, NUSAP1 and TK1 was compared between normal human liver and HCC tissues using the Oncomine database. The Human Protein Atlas database was also used to verify the protein expression levels of these genetic markers. The analysis of a representative dataset (Roessler Liver 2) revealed that CCNB2, NUSAP1 and TK1 mRNA expression was maintained at significantly higher levels in HCC vs. normal liver tissues, which was consistent with the microarray results. The expression of the 3 genetic markers in HCC determined in the above analysis was also consistent with the histological evaluation results from the Human Protein Atlas database (Fig. 6). Furthermore, RT-qPCR was used to verify the expression levels of the 3 genetic markers in primary HCC and paired adjacent tissues. In the 16 HCC tissues, CCNB2, NUSAP1 and TK1 were significantly upregulated as compared with those in their matched adjacent tissues $(\mathrm{P}<0.001 ;$ Fig. 7A-C). In addition, a highly positive correlation was determined between the expression of CCNB2 and NUSAP1 $(\mathrm{P}<0.05)$, while the expression of CCNB2 and TK1 and that of NUSPA1 and TK1 exhibited a moderate positive correlation $(\mathrm{P}<0.05$; Fig. 7D-F).

\section{Discussion}

In the present study, DEGs from 3 GEO datasets were screened. A total of 96 DEGs were identified following RRA analysis, including 25 up- and 71 downregulated genes. Following enrichment and PPI analysis, 15 genes were identified as hub genes. Among these 15 hub genes, CCNB2, NUSAP1 and TK1 were closely correlated with one another, and may participate in the E2F1 pathway interacting with the BRCA1 pathway. It was also indicated that these genes may be involved in HCC progression.

DEG enrichment analysis suggested that 'anaphasepromoting complex-dependent catabolic process', 'oxidation-reduction process' and 'cell division' were the top 3 most significantly enriched BPs. The major reason for tumorigenesis may be cell cycle imbalance, which then induces excessive cell proliferation. Furthermore, several of the 15 hub genes were indicated to be involved in cancer cell growth and survival. Targeting of AURKA by inhibitor alisertib potently induced apoptosis in HCC cells, which was consistent with the results of clinical trials for hematological malignancies and solid tumors $(19,20)$. CDC20 has been reported to be closely associated with the tumorigenesis and progression of multiple cancers (21). TOP2A overexpression is implicated in early-stage HCC development, short survival and chemoresistance in patients with $\operatorname{HCC}(22,23)$. $\mathrm{KIFC1}$ is considered a potential biomarker for non-small cell lung cancer and may have a significant reference value for ovarian adenocarcinoma metastasis $(24,25)$. Increased KIFC1 mRNA expression in HCC induced cell metastasis through the G-ankyrin/AKT/TWIST1 signaling pathway (26). PRC1 was not only overexpressed in several types of cancer, but it was also reported to enhance early HCC development through the Wnt/ $\beta$-catenin pathway (27-29). KIF20A is a downstream target of Hedgehog signaling and crucial to the growth of human HCC cells through the GLI family zinc finger 2/KIF20A axis $(30,31)$. RACGAP1 was reported to be highly upregulated in multiple types of cancer $(32,33)$. A recent study also suggested that RACGAP1 was essential for HCC cell cytokinesis and suppressed the Hippo/yes-associated protein-1 pathway (34). PTTG1 is closely associated with hepatitis $B$ virus $\mathrm{x}(\mathrm{HBx})$ protein and was indicated to be highly expressed in HBx-immunoreactive cells, which may contribute to cirrhosis and $\mathrm{HCC}$ in patients with chronic hepatitis B $(35,36)$. The expression of CDCA5 was reported to be closely linked to microvascular invasion and tumor diameter, and inhibition of CDCA5 expression impeded hepatoma cell proliferation and induced apoptosis (37). UBE2C was indicated 

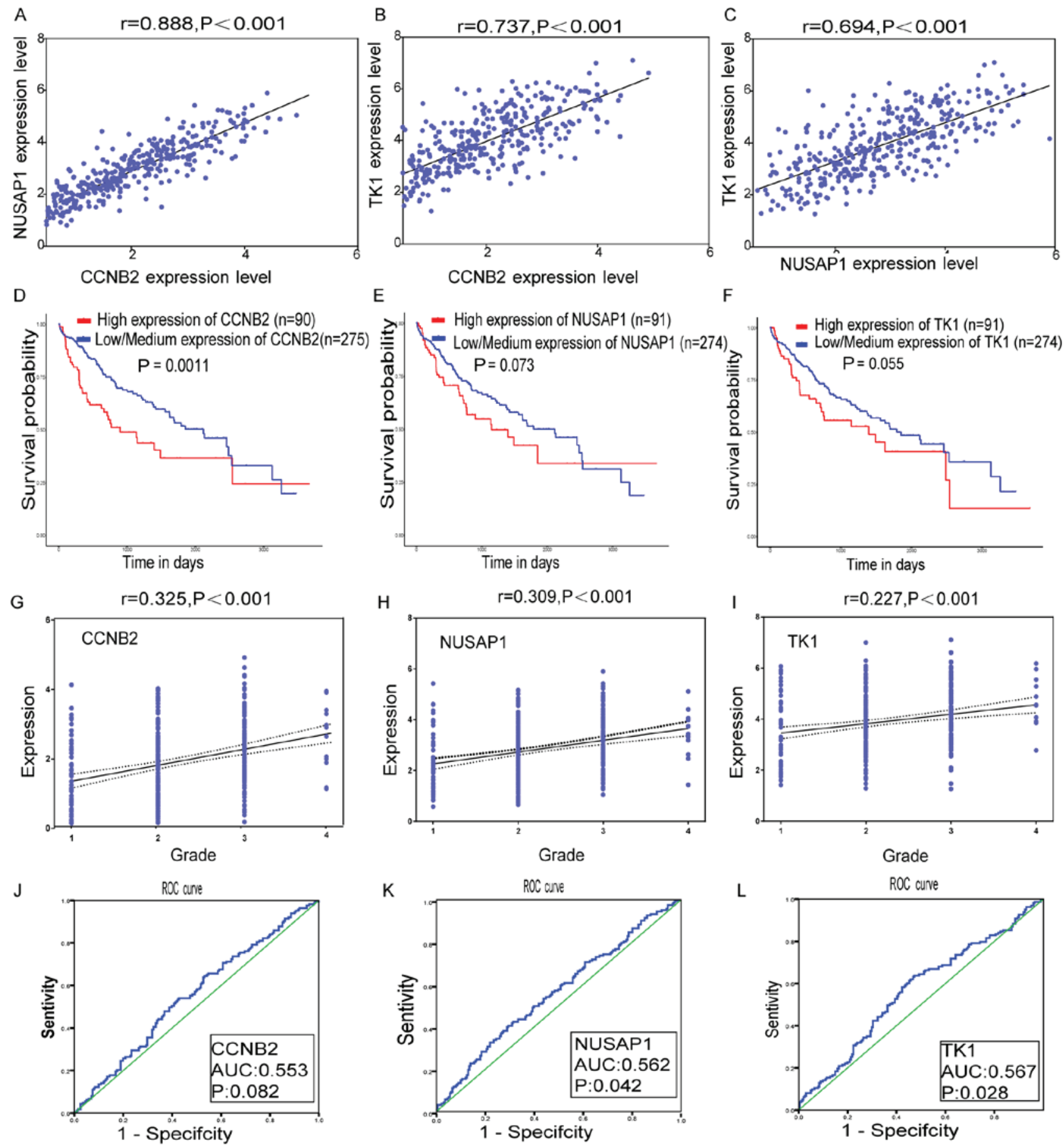

Figure 5. (A-C) CCNB2, NUSAP1 and TK1 levels were highly correlated to one another (data from TCGA). (D-F) Survival analysis of genetic markers in the TCGA datasets. (D) CCNB2, (E) NUSAP1 and (F) TK1. Red lines represent high expression of the genes and blue lines represent low/medium expression. (G-I) Correlation between the expression levels of genetic markers in the TCGA dataset and the disease progression of HCC. (G) CCNB2, (H) NUSAP1, (I) TK1. Grade means histological grade. (J-L) ROC analysis of genetic markers in TCGA database. (J) CCNB2, (K) NUSAP1 and (L) TK1. ROC curves and AUC statistics to evaluate the capacity of the distinguishing recurrent and non-recurrent hepatocellular carcinoma are provided. CCNB2, cyclin B2; NUSAP1, nucleolar and spindle-associated protein 1; TK1, thymidine kinase 1; TCGA, The Cancer Genome Atlas; ROC, receiver operating characteristic; AUC, area under curve.

to be highly expressed in HCC tumor tissues and patients with upregulated UBE2C were more likely to have higher-grade tumors and poor prognosis $(38,39)$. Overexpression of MELK leads to cell cycle- and mitosis-associated gene expression, which influences cell division. In addition, MELK was defined as an oncogenic kinase in early HCC recurrence $(40,41)$. To date, the function of CKAP2L in cancer has rarely been investigated and its mechanism has remained elusive $(42,43)$.
Besides the hub genes mentioned above, the present study focused on the CCNB2, NUSAP1 and TK1 genes, and PPI analysis suggested that CCNB2, NUSAP1 and TK1 are closely associated with other hub genes. BRCA1 was indicated to be one of the protein targets of double-stand breaks and ATM/ATR and E2F1 was involved in the cell cycle by influencing the formation of RAD51 foci, which was controlled by BRCA1 and BRCA2 (44), and BRCA1 
CCNB2
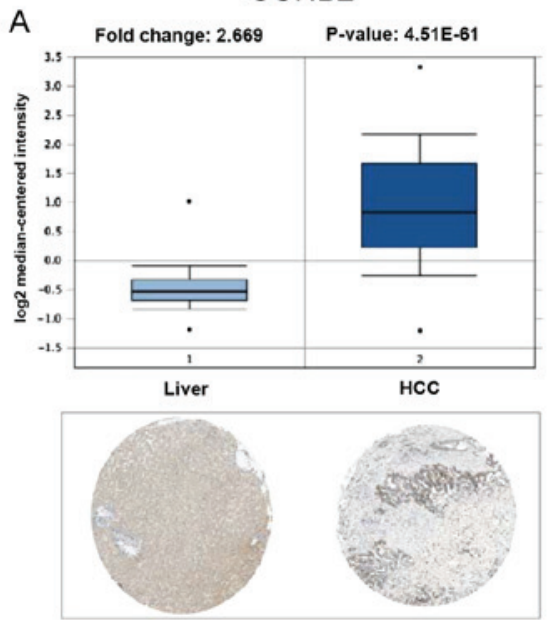

NUSAP1

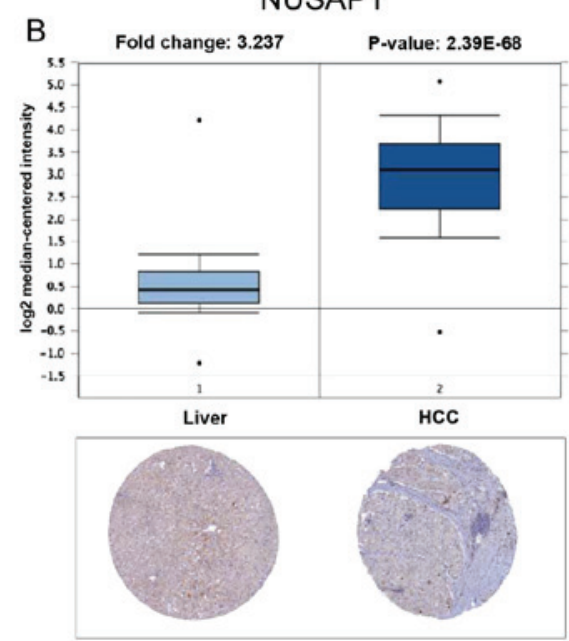

TK1

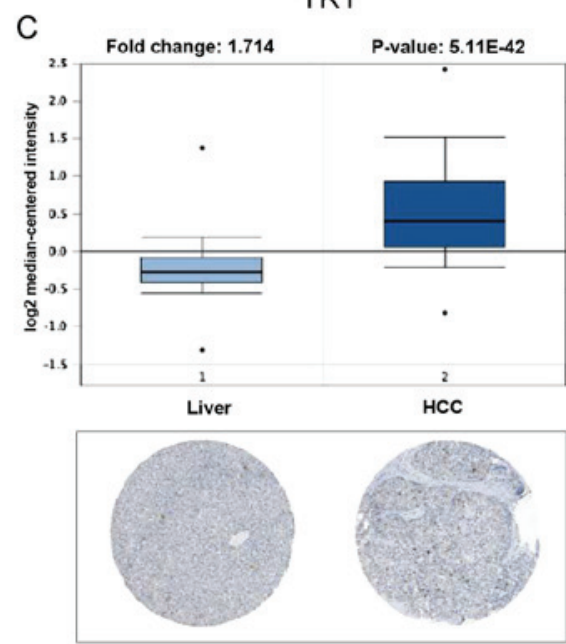

Figure 6. Validation of the expression of three genes at the transcriptional and translational level by Oncomine database and The Human Protein Atlas database (immunohistochemistry). (A) CCNB2, (B) NUSAP1 and (C) TK1. Magnification, x100. CCNB2, cyclin B2; NUSAP1, nucleolar and spindle-associated protein 1; TK1, thymidine kinase 1 ; HCC, hepatocellular carcinoma.

A

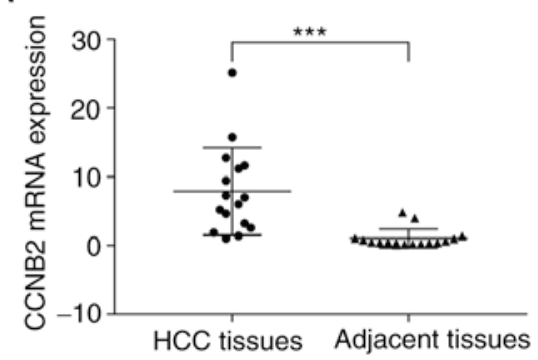

$\mathrm{D}$

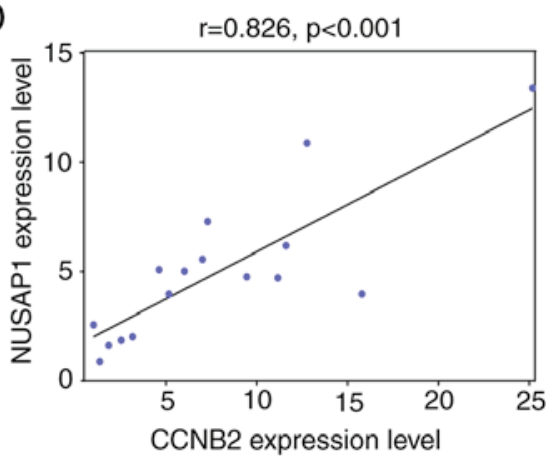

$\mathrm{B}$

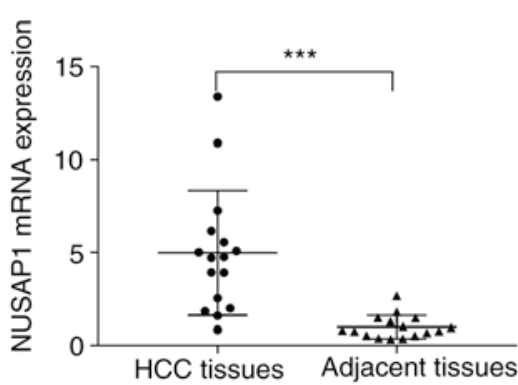

$\mathrm{E}$

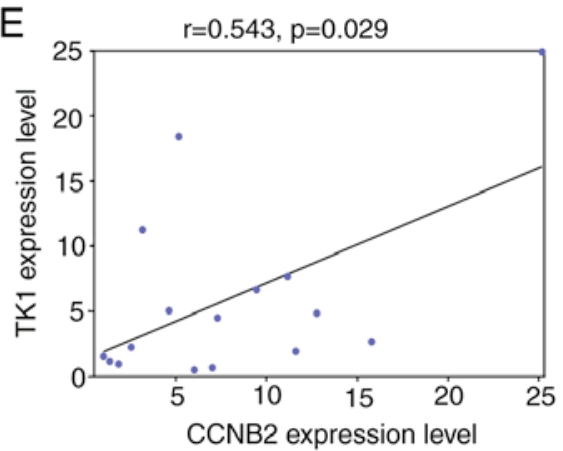

C

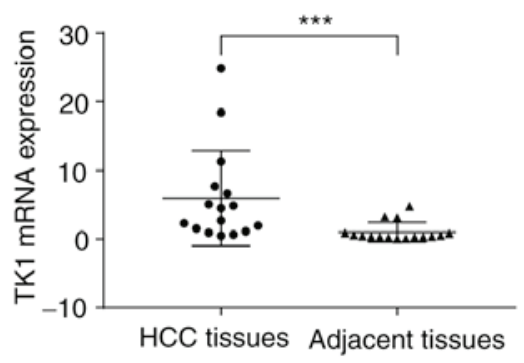

$\mathrm{F}$

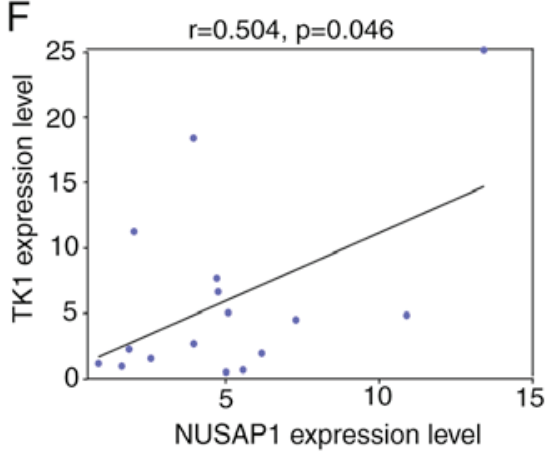

Figure 7. Overexpression of markers in HCC. (A) CCNB2, (B) NUSAPA1 and (C) TK1 ( $\mathrm{n}=16$ ). ${ }^{* * *} \mathrm{P}<0.001$ compared with the adjacent tissues. (D-F) CCNB2, NUSAP1 and TK1 levels were highly correlated with one another $(\mathrm{n}=16)$. CCNB2, cyclin B2; NUSAP1, nucleolar and spindle-associated protein 1; TK1, thymidine kinase 1 ; HCC, hepatocellular carcinoma.

has two splice variants, BRCA1a and BRCA1b, which are able to associate with E2F transcriptional factors, including cyclins/cyclin D kinase (CDK) to regulate the cell cycle (45). In a previous study, based on integration of GRNInfer with GO, KEGG, BioCarta, GNF-U133A, UNIGENE-EST, Disease and GenMAPP databases by DAVID and MAS 3.0, CCNB2, NUSAP1 and TK1 were key molecules in a novel hypothetic mechanism of the E2F1 interacting with the BRCA1 pathway, where CCNB2 and NUSAP1 were upstream molecules and TK1 an important downstream molecule (18). CCNB2 belongs to the B-type cyclin family and is known to activate CDKs during the cell cycle; inhibition of the expression of CCNB2 in BEL-7404 cells was reported to decrease cell proliferation and migration, increase cell apoptosis and induce $S$-phase arrest $(46,47)$. NUSAP1 is a $55-\mathrm{kDa}$ protein involved in cell proliferation, which interacts with microtubules and causes chromosome segregation. NUSAP1 has a key role in BRCA1-regulated pathways, as it regulates BRCA1 protein levels from the early S-phase into G2-phase (48). There are two CCAAT boxes and binding sites for E2F1 in the promoter region of NUSAP and overexpression of E2F1 results in increased 
expression of NUSAP; NUSAP overexpression appears to be driven in part by E2F1 activation (49). TK1 is involved in DNA repair and is upregulated during $\mathrm{S}$-phase. A study on pancreatic ductal adenocarcinoma suggested that patients with upregulated TK1 had poor prognosis and that the transcription factor E2F1 may lead to tumor cell proliferation by regulating TK1 expression (50-53).

In the present study, further analysis using data from TCGA indicated a close correlation among CCNB2, NUSAP1 and TK1. CCNB2 was significantly associated with the survival of patients with HCC. The ROC curve and calculated AUC suggested that NUSAP1 and TK1 were capable of distinguishing between recurrent and non-recurrent HCC. In addition, CCNB2, NUSAP1 and TK1 were all highly correlated with the HCC grade. Furthermore, the Oncomine database and the HPA database were used to verify the transcriptional and translational expression levels of the 3 genes. It was revealed that, as compared with normal tissue, the 3 genes were significantly upregulated in HCC. In addition, RT-qPCR demonstrated that the mRNA expression of CCNB2, NUSAP1 and TK1 was increased in 16 primary HCC tissues as compared with that in the matched adjacent tissues, and the 3 genes were closely correlated with one another. The increase in CCNB2 and NUSAP1 expression was highly correlated with the expression of TK1, and based on the Kaplan Meier analysis, high expression levels of TK1 was linked to poor prognosis, indicating that it may be more accurate if the combination of CCNB2. While NUSAP1 and TK1 is used as a prognostic marker for $\mathrm{HCC}$, the detailed mechanism of CCNB2-NUSAP1-TK1 on the cell cycle and apoptosis of HCC cells warrants further investigation.

Only a few studies have focused on the association between these 3 genes and their role in the E2F1 pathway interacting with the BRCA1 pathway. This was attempted in the present study. The present results demonstrated that CCNB2, NUSAP1 and TK1 were significantly upregulated in HCC tissues compared with adjacent normal tissues through RT-qPCR analysis, as well as bioinformatics analyses of the TCGA and GEO databases. High expression levels of CCNB2, NUSAP1 and TK1 in HCC tissues were associated with poor prognosis and high risk of reccurrence. However, further studies are required to explore the biological functions of these 3 genes in HCC and/or other cancer types, including metabolism and cell cycle. This may indicate their utility as targets in the treatment of HCC and/or other cancer types and provide details on the interactions between them. The roles of the other 12 hub genes that do not interact with E2F1 and the BRCA1 pathway in $\mathrm{HCC}$ also require further investigation.

In conclusion, using a series of rigorous bioinformatics analyses, it was indicated that the expression levels of CCNB2, NUSAP1 and TK1 were not only closely correlated with the HCC grade but also associated with OS, and the expression of the 3 genes at the transcriptional and translational level also proved their vital role in HCC. These genes not only regulate key steps of the cell cycle and catabolic processes, targeting individual genes and potentially affecting cancer cell development and growth, but are also closely associated with one another. CCNB2, NUSAP1 and TK1 may therefore be reliable prognostic biomarkers for HCC, and even as possible oncogenes and therapeutic targets in HCC.
In the present study, bioinformatics analyses were used to screen for DEGs between HCC and non-tumor tissues. Among the 15 hub genes, CCNB2, NUSAP1 and TK1 were indicated to be correlated with one another, and those genes were indicated to be able to help predict the prognosis of HCC patients. The results of the present study may prove valuable from the perspectives of basic research and clinical treatment for HCC. However, even though the present results may contribute to the understanding of the progression of $\mathrm{HCC}$, further studies are required to verify genes associated with HCC.

\section{Acknowledgements}

Not applicable.

\section{Funding}

This research was funded by the National Natural Science Foundation of China (grant nos. 81572994 and 81872491), Guangxi Science and Technology Base and Talent Special Project (grant no. 2017AD10045), Key Laboratory of the Ministry of Education Project for Early Prevention and Treatment of Regional High-risk Tumors (grant no. GKE2018-03) and the Guangxi Nanning Qing Xiu District Science and Technology Research and Technology Project (grant no. 2014S03).

\section{Availability of data and materials}

The datasets used and/or analyzed during the present study are available from the corresponding author on reasonable request.

\section{Author's contributions}

LL and AC were involved in drafting the manuscript, design of the study, acquisition and analysis of data and approval of the final version to be published. SC, WS, QY and PW contributed to the conception and design of the study and the acquisition of the data. SZ was responsible for analysis and interpretation of the data. All authors read and approved the final manuscript.

\section{Ethics approval and consent to participate}

The present study was approved by the Ethics Committee of Guangxi Medical University (Guangxi, China) and in accordance with the Guangxi Medical University guidelines and regulations. The patients provided written informed consent; furthermore, the authors made efforts to remove any identifying information to protect the privacy of the patients.

\section{Patient consent for publication}

Not applicable.

\section{Competing interests}

The authors declare that they have no competing interests. 


\section{References}

1. Siegel RL, Miller KD and Jemal A: Cancer statistics, 2019. CA Cancer J Clin 69: 7-34, 2019.

2. Villanueva A: Hepatocellular carcinoma. N Engl J Med 380: $1450-1462,2019$.

3. Bertuccio P, Turati F, Carioli G, Rodriguez T, La Vecchia C, Malvezzi M and Negri E: Global trends and predictions in hepatocellular carcinoma mortality. J Hepatol 67: 302-309, 2017.

4. Mak LY, Cruz-Ramón V, Chinchilla-López P, Torres HA, LoConte NK, Rice JP, Foxhall LE, Sturgis EM, Merrill JK, Bailey $\mathrm{HH}$, et al: Global epidemiology, prevention, and management of hepatocellular carcinoma. Am Soc Clin Oncol Educ Book 38: 262-279, 2018.

5. Bosetti C, Turati F and La Vecchia C: Hepatocellular carcinoma epidemiology. Best Pract Res Clin Gastroenterol 28: 753-770, 2014.

6. Turati F, Galeone C, Rota M, Pelucchi C, Negri E, Bagnardi V, Corrao G, Boffetta P and La Vecchia C: Alcohol and liver cancer: A systematic review and meta-analysis of prospective studies. Ann Oncol 25: 1526-1535, 2014.

7. Jindal A, Thadi A and Shailubhai K: Hepatocellular carcinoma: Etiology and current and future drugs. J Clin Exp Hepatol 9: 221-232, 2019.

8. Bedard PL, Hansen AR, Ratain MJ and Siu LL: Tumour heterogeneity in the clinic. Nature 501: 355-364, 2013.

9. Ward PS and Thompson CB: Metabolic reprogramming: A cancer hallmark even warburg did not anticipate. Cancer Cell 21: 297-308, 2012.

10. Pavlova NN and Thompson CB: The emerging hallmarks of cancer metabolism. Cell Metab 23: 27-47, 2016.

11. Dimri M, Humphries A, Laknaur A, Elattar S, Lee TJ, Sharma A, Kolhe R and Satyanarayana A: NAD $(\mathrm{P}) \mathrm{H}$ quinone dehydrogenase 1 ablation inhibits activation of the phosphoinositide 3-kinase/Akt Serine/threonine kinase and mitogen-activated protein kinase/extracellular signal-regulated kinase pathways and blocks metabolic adaptation in hepatocellular carcinoma. Hepatology: Jun 19, 2019 (Epub ahead of print). doi: 10.1002/hep.30818.

12. Liu Y, Gong W, Yang ZY, Zhou XS, Gong C, Zhang TR, Wei X, Ma D, Ye F and Gao QL: Quercetin induces protective autophagy and apoptosis through ER stress via the p-STAT3/Bcl-2 axis in ovarian cancer. Apoptosis 22: 544-557, 2017.

13. Wu L, Li J, Liu T, Li S, Feng J, Yu Q, Zhang J, Chen J, Zhou Y, Ji J, et al: Quercetin shows anti-tumor effect in hepatocellular carcinoma LM3 cells by abrogating JAK2/STAT3 signaling pathway. Cancer Med 8: 4806-4820, 2019.

14. Anastas JN and Moon RT: WNT signalling pathways as therapeutic targets in cancer. Nat Rev Cancer 13: 11-26, 2013.

15. Uhlén M, Fagerberg L, Hallström BM, Lindskog C, Oksvold P, Mardinoglu A, Sivertsson Å, Kampf C, Sjöstedt E, Asplund A, et al: Proteomics. Tissue-based map of the human proteome. Science 347: 1260419, 2015.

16. Chandrashekar DS, Bashel B, Balasubramanya SAH, Creighton CJ, Ponce-Rodriguez I, Chakravarthi BVSK and Varambally S: UALCAN: A portal for facilitating tumor subgroup gene expression and survival analyses. Neoplasia 19: 649-658, 2017.

17. Livak KJ and Schmittgen TD: Analysis of relative gene expression data using real-time quantitative PCR and the 2(-Delta Delta C(T)) method. Methods 25: 402-408, 2001.

18. Chen Q, Wang L, Jiang M, Huang J, Jiang Z, Feng H and Ji Z: E2F1 interactive with BRCA1 pathway induces HCC two different small molecule metabolism or cell cycle regulation via mitochondrion or CD4 ${ }^{+} \mathrm{T}$ to cytosol. J Cell Physiol 233: 1213-1221, 2018.

19. Goldenson B and Crispino JD: The aurora kinases in cell cycle and leukemia. Oncogene 34: 537-545, 2015.

20. Schneider MA, Christopoulos P, Muley T, Warth A, Klingmueller U, Thomas M, Herth FJ, Dienemann H, Mueller NS, Theis F and Meister M: AURKA, DLGAP5, TPX2, KIF11 and CKAP5: Five specific mitosis-associated genes correlate with poor prognosis for non-small cell lung cancer patients. Int J Oncol 50: 365-372, 2017.

21. Chu Z, Zhang X, Li Q, Hu G, Lian CG and Geng S: CDC20 contributes to the development of human cutaneous squamous cell carcinoma through the $\mathrm{Wnt} / \beta$-catenin signaling pathway. Int J Oncol 54: 1534-1544, 2019.

22. Nitiss JL: DNA topoisomerase II and its growing repertoire of biological functions. Nat Rev Cancer 9: 327-337, 2009.
23. Wong N, Yeo W, Wong WL, Wong NL, Chan KY, Mo FK, Koh J, Chan SL, Chan AT, Lai PB, et al: TOP2A overexpression in hepatocellular carcinoma correlates with early age onset, shorter patients survival and chemoresistance. Int J Cancer 124: 644-652, 2009.

24. Yu K, Hou L, Zhu JQ, Ying XP and Yang WX: KIFC1 participates in acrosomal biogenesis, with discussion of its importance for the perforatorium in the Chinese mitten crab Eriocheir sinensis. Cell Tissue Res 337: 113-123, 2009.

25. Pawar S, Donthamsetty S, Pannu V, Rida P, Ogden A, Bowen N, Osan R, Cantuaria G and Aneja R: KIFCI, a novel putative prognostic biomarker for ovarian adenocarcinomas: Delineating protein interaction networks and signaling circuitries. J Ovarian Res 7: 53, 2014

26. Han J, Wang F, Lan Y, Wang J, Nie C, Liang Y, Song R, Zheng T, Pan S, Pei T, et al: KIFC1 regulated by miR-532-3p promotes epithelial-to-mesenchymal transition and metastasis of hepatocellular carcinoma via gankyrin/AKT signaling. Oncogene 38: 406-420, 2019.

27. Chen J, Rajasekaran M, Xia H, Zhang X, Kong SN, Sekar K, Seshachalam VP, Deivasigamani A, Goh BK, Ooi LL, et al: The microtubule-associated protein PRC1 promotes early recurrence of hepatocellular carcinoma in association with the Wnt/ $\beta$-catenin signalling pathway. Gut 65: 1522-1534, 2016.

28. Subramanian R, Wilson-Kubalek EM, Arthur CP, Bick MJ, Campbell EA, Darst SA, Milligan RA and Kapoor TM: Insights into antiparallel microtubule crosslinking by PRC1, a conserved nonmotor microtubule binding protein. Cell 142: 433-443, 2010.

29. Wang SM, Ooi LL and Hui KM: Upregulation of Rac GTPase-activating protein 1 is significantly associated with the early recurrence of human hepatocellular carcinoma. Clin Cancer Res 17: 6040-6051, 2011.

30. Verhey KJ and Hammond JW: Traffic control: Regulation of kinesin motors. Nat Rev Mol Cell Biol 10: 765-777, 2009.

31. Shi C, Huang D, Lu N, Chen D, Zhang M, Yan Y, Deng L, Lu Q, $\mathrm{Lu} \mathrm{H}$ and Luo S: Aberrantly activated Gli2-KIF20A axis is crucial for growth of hepatocellular carcinoma and predicts poor prognosis. Oncotarget 7: 26206-26219, 2016.

32. Imaoka H, Toiyama Y, Saigusa S, Kawamura M, Kawamoto A, Okugawa Y, Hiro J, Tanaka K, Inoue Y, Mohri Y and Kusunoki M: RacGAP1 expression, increasing tumor malignant potential, as a predictive biomarker for lymph node metastasis and poor prognosis in colorectal cancer. Carcinogenesis 36: 346-354, 2015.

33. Saigusa S, Tanaka K, Mohri Y, Ohi M, Shimura T, Kitajima T, Kondo S, Okugawa Y, Toiyama Y, Inoue Y and Kusunoki M: Clinical significance of RacGAP1 expression at the invasive front of gastric cancer. Gastric Cancer 18: 84-92, 2015.

34. Yang XM, Cao XY, He P, Li J, Feng MX, Zhang YL, Zhang XL, Wang YH, Yang Q, Zhu L, et al: Overexpression of Rac GTPase activating protein 1 contributes to proliferation of cancer cells by reducing hippo signaling to promote cytokinesis. Gastroenterology 155: 1233-1249 e22, 2018.

35. Zou H, McGarry TJ, Bernal T and Kirschner MW: Identification of a vertebrate sister-chromatid separation inhibitor involved in transformation and tumorigenesis. Science 285: 418-422, 1999.

36. Molina-Jiménez F, Benedicto I, Murata M, Martin-Vilchez S, Seki T, Antonio Pintor-Toro J, Tortolero M, Moreno-Otero R, Okazaki K, Koike K, et al: Expression of pituitary tumor-transforming gene 1 (PTTG1)/securin in hepatitis B virus (HBV)-associated liver diseases: Evidence for an HBV $\mathrm{X}$ protein-mediated inhibition of PTTG1 ubiquitination and degradation. Hepatology 51: 777-787, 2010.

37. Tian Y, Wu J, Chagas C, Du Y, Lyu H, He Y, Qi S, Peng Y and $\mathrm{Hu} \mathrm{J}$ : CDCA5 overexpression is an Indicator of poor prognosis in patients with hepatocellular carcinoma (HCC). BMC Cancer 18: 1187, 2018.

38. Rape M and Kirschner MW: Autonomous regulation of the anaphase-promoting complex couples mitosis to S-phase entry. Nature 432: 588-595, 2004.

39. Fernandez Esmerats J, Villa-Roel N, Kumar S, Gu L, Salim MT, Ohh M, Taylor WR, Nerem RM, Yoganathan AP and Jo H: Disturbed flow increases UBE2C (Ubiquitin E2 Ligase C) via loss of miR-483-3p, inducing aortic valve calcification by the pVHL (von Hippel-Lindau protein) and HIF-1 $\alpha$ (hypoxia-inducible factor-1 $\alpha$ ) pathway in endothelial cells. Arterioscler Thromb Vasc Biol 39: 467-481, 2019.

40. Xia H, Kong SN, Chen J, Shi M, Sekar K, Seshachalam VP, Rajasekaran M, Goh BKP, Ooi LL and Hui KM: MELK is an oncogenic kinase essential for early hepatocellular carcinoma recurrence. Cancer Lett 383: 85-93, 2016. 
41. Blot J, Chartrain I, Roghi C, Philippe M and Tassan JP: Cell cycle regulation of $\mathrm{pEg} 3$, a new Xenopus protein kinase of the KIN1/PAR-1/MARK family. Dev Biol 241: 327-338, 2002.

42. Hussain MS, Battaglia A, Szczepanski S, Kaygusuz E, Toliat MR Sakakibara S, Altmüller J, Thiele H, Nürnberg G, Moosa S, et al: Mutations in CKAP2L, the human homolog of the mouse Radmis gene, cause Filippi syndrome. Am J Hum Genet 95: 622-632, 2014.

43. Xiong G, Li L, Chen X, Song S, Zhao Y, Cai W and Peng J: Up-regulation of CKAP2L expression promotes lung adenocarcinoma invasion and is associated with poor prognosis. Onco Targets Ther 12: 1171-1180, 2019.

44. Chen J,Zhu F, Weaks RL, Biswas AK, Guo R, Li Y and Johnson DG: E2F1 promotes the recruitment of DNA repair factors to sites of DNA double-strand breaks. Cell Cycle 10: 1287-1294, 2011.

45. Wang H, Shao N, Ding QM, Cui J, Reddy ES and Rao VN: BRCA1 proteins are transported to the nucleus in the absence of serum and splice variants BRCA1a, BRCA1b are tyrosine phosphoproteins that associate with $\mathrm{E} 2 \mathrm{~F}$, cyclins and cyclin dependent kinases. Oncogene 15: 143-157, 1997.

46. Li R, Jiang X, Zhang Y, Wang S, Chen X, Yu X, Ma J and Huang X: Cyclin B2 overexpression in human hepatocellular carcinoma is associated with poor prognosis. Arch Med Res 50: 10-17, 2019.

47. Wu T, Zhang X, Huang X, Yang Y and Hua X: Regulation of cyclin B2 expression and cell cycle $\mathrm{G} 2 / \mathrm{m}$ transition by menin. J Biol Chem 285: 18291-18300, 2010.

48. Raemaekers T, Ribbeck K, Beaudouin J, Annaert W, Van Camp M, Stockmans I, Smets N, Bouillon R, Ellenberg J and Carmeliet G: NuSAP, a novel microtubule-associated protein involved in mitotic spindle organization. J Cell Biol 162: 1017-1029, 2003.
49. Gulzar ZG, McKenney JK and Brooks JD: Increased expression of NuSAP in recurrent prostate cancer is mediated by E2F1. Oncogene 32: 70-77, 2013.

50. Zhu X, Shi C, Peng Y, Yin L, Tu M, Chen Q, Hou C, Li Q and Miao Y: Thymidine kinase 1 silencing retards proliferative activity of pancreatic cancer cell via E2F1-TK1-P21 axis. Cell Prolif 51: e12428, 2018.

51. Mao R, Liu J, Liu G, Jin S, Xue Q, Ma L, Fu Y, Zhao N, Xing J, $\mathrm{Li} \mathrm{L}$, et al: Whole genome sequencing of matched tumor, adjacent non-tumor tissues and corresponding normal blood samples of hepatocellular carcinoma patients revealed dynamic changes of the mutations profiles during hepatocarcinogenesis. Oncotarget 8: 26185-26199, 2017.

52. Kauffman MG and Kelly TJ: Cell cycle regulation of thymidine kinase: Residues near the carboxyl terminus are essential for the specific degradation of the enzyme at mitosis. Mol Cell Biol 11: 2538-2546, 1991

53. Ke PY and Chang ZF: Mitotic degradation of human thymidine kinase 1 is dependent on the anaphase-promoting complex/cyclosome-CDH1-mediated pathway. Mol Cell Biol 24: 514-526, 2004.

This work is licensed under a Creative Commons Attribution-NonCommercial-NoDerivatives 4.0 International (CC BY-NC-ND 4.0) License. 